\title{
A Handoff Algorithm of Dynamic Decisions with Extenics for Wireless Cellular Networks
}

\author{
Chen-Feng Wu \\ Department of Information Management, Yu Da University, 168, Hsueh-fu Road, Tanwen Village, Chaochiao Township, \\ Miaoli County 361, Taiwan \\ Correspondence should be addressed to Chen-Feng Wu, cfwu@ydu.edu.tw
}

Received 10 June 2009; Accepted 12 January 2010

Academic Editor: Theodoros Tsiftsis

Copyright (๑) 2010 Chen-Feng Wu. This is an open access article distributed under the Creative Commons Attribution License, which permits unrestricted use, distribution, and reproduction in any medium, provided the original work is properly cited.

A traditional handoff algorithm always adopts stationary channel preservation schemes that cause the increase of call blocking probability (CBP) and the decrease of system utilization to reduce the call dropping probability (CDP) for handoff call. In this paper, the extension theory is introduced and applied to the handoff algorithm for participating handoff decisions and channel preservation. The mobile can perform handoff by predicting user mobility in advance based on extension-based derivation. By using the simple extension-based derivation, the required channels can be reserved for handoff call beforehand, and the required quality of service (QoS) is preserved during handoff occurring. Furthermore, both the QoS issue and the efficiency of channel allocation are considered simultaneously for the proposed algorithm. The extension and accumulation concepts are combined for reducing the number of guard channels in the proposed scheme, so the CBP does not reduce too much and the utilization of channel is more efficiency.

\section{Introduction}

Due to the rapid evolution of mobile networks, the corresponding applications are also varied from low-speed voice data to high-speed multimedia services. The most important issue is to support quality of service (QoS) for such large number of required bandwidth to multimedia services in wireless cellular networks. It is difficult to allocate suitable bandwidth for mobiles before handoff to the appropriate cell owe to limited wireless radio spectrum. Because of the variable user mobility, it became more complex to predict the appropriate cell for handoff. Thus, user mobility analysis became a dominant fact to tell whether an effective handoff algorithm is or not. An effective handoff algorithm, however, should not only take quality of service (QoS) into account, but also consider the blocking probability of a new call and resource utilization simultaneously. Therefore, supporting QoS requires careful management of wireless bandwidth.

There have been so many studies [1-3] proposed for QoS guarantee as well as effective user mobility and resource management have been also proposed in [4]. In general, the literatures always think the improvement of bandwidth is a dynamic channel allocation (DCA) problem, and the reservation schemes were also proposed for reducing call dropping rate (CDR). However, seldom literature has developed to satisfy both issues at the same time. Prioritized channel assignment schemes for accommodating handoff attempts and optimization studies for assigning channels to priority classed have been proposed in the literature $[5,6]$. Whatever, user mobility has been regarded as a crucial factor for affecting traffic performance in cellular networks.

Thus, there have been many studies focused on the prediction of user mobility for guaranteeing QoS in cellular networks. Although the decrease of CDR is the most important issue for QoS in cellular networks, the resource can not be sacrificed too much for achieving QoS goal that may causes the inefficient resource utilization. The emergency problem of extensive demand of bandwidth for multimedia services in cellular networks is to guarantee QoS and to make the resource utilization efficiency. The resource utilization must be taken into consideration when the resource is allocated for handoffs by predicting user 
mobility. Therefore, a proper handoff algorithm that can reserve the required bandwidth for which cell the movement is predicted should be proposed. In this paper, a handoff algorithm based on extenics derivation is proposed to predict the movement of mobile and to calculate the accumulated attempts of the adjacent cell to which the mobile potentially handoff by combining the extenics theory, user regular routines information, and the speed of mobile movement.

\section{Preliminary and System Model}

There are many existing unsolvable problems that can be changed into solvable through some methods of transformation in the real word. For example, it is impossible for utilizing a steelyard to weigh an elephant. However, the wellknown story about "Tsao Chung weighs an elephant" that revealed a possible way of solving this weighing problem happened in ancient China. Therefore, there has been an interest in the research of Extension Engineering, which is motivated by the potential benefits of Extension Set Theory. The Extension Set Theory, called Extenics [7], is developed for solving conflicts in problems or providing equivalent solutions. Problems are solved with experiences by human, some of them can be applied with straightforward thoughts, but there still leaves conflicts and incompatible parts that cannot be solved. Equivalent solutions are needed to transform the original problem to another such as Laplace Transform that is a good example. In our proposed method the extended relational function and extension set are used to define the threshold dynamically for the speed of mobile movement.

The original relational function was adjusted for defining the speed of mobile movement in cellular networks. There are three kinds of movement speed defined in Table 1. The probability of handoff is very low except only just along the edge of two cells when a pedestrian take a walk around no matter urban area or suburban area. In order to include the situation of walking along the edge of two cells, the definition value of relational function is modified to be 0.1 . In contrast to pedestrian, vehicles mobility can be classified two classes; one is that vehicles move in the speed of $5 \mathrm{~km} / \mathrm{hr}$ to $50 \mathrm{~km} / \mathrm{hr}$ around urban area, and the other is that vehicles move in the speed of faster than $50 \mathrm{~km} / \mathrm{hr}$ around suburbs. The first case can be regarded as extension part for extenics, because the speed of vehicles may be slower than pedestrian for a traffic jam and faster than $50 \mathrm{~km} / \mathrm{hr}$ when the traffic is smooth. Thus the definition value of function can be defined as $k(v)=(v-5) /(50-5)$. In the other case, because the vehicles move in the speed of faster than $55 \mathrm{~km} / \mathrm{hr}$, the probability of handoff occur will be very high.

The proposed scheme is suitable for the traditional honeycomb structure, so the potential cells of handoff just only six adjacent ones. For example, illustrated in Figure 1, cell "a" may handoff to the cell that is adjacent such as cell "b", “c”, “d”, “e”, "f”, or "g”. Thus, the necessarily considered cells for the handoff algorithm are only six ones for the honeycomb cell structure, so the proposed scheme regards cell "a" as an initial point then to calculate the potential
TABLE 1: Definition of relational function.

\begin{tabular}{|c|c|c|}
\hline Speed range & $\begin{array}{l}\text { Potential category of } \\
\text { traffic }\end{array}$ & $\begin{array}{l}\text { Definition of modified } \\
\text { relational function } k(v)\end{array}$ \\
\hline Speed $<5 \mathrm{~km} / \mathrm{hr}$ & Pedestrians & $k(v)=0.1$ \\
\hline $\begin{array}{l}5 \mathrm{~km} / \mathrm{hr}<\text { Speed } \\
<50 \mathrm{~km} / \mathrm{hr}\end{array}$ & $\begin{array}{l}\text { Vehicles in urban } \\
\text { area }\end{array}$ & $k(v)=(v-5) /(50-5)$ \\
\hline$>50 \mathrm{~km} / \mathrm{hr}$ & Vehicles in suburbs & $k(v)=1$ \\
\hline
\end{tabular}

TABLE 2: Information of cell for each mobile

\begin{tabular}{lc}
\hline Current cell number & $(i)$ \\
Average dwell time & $T_{\text {dwell_avg }}$ \\
Minimum dwell time & $T_{\text {dwell_min }}$ \\
Adjacent Cell No & $(j)$ \\
$\begin{array}{l}\text { Probability of movement } \\
\text { (where } j \text { is the adjacent cell) }\end{array}$ & $P_{\text {move }(j)}$ \\
\hline
\end{tabular}

handoff attempts that are defined by accumulation function for each adjacent cell. In order to define the accumulation function, we find out that the affecting factors that are movement speed and user regular routes are discovered for handoffs. The movement speed is defined by the relational function of Extenics [7], and the potential probability of movement for each cell can be obtained by analyzing user regular routes. To obtain the movement probability for each mobile in every cell, the proposed scheme reduces the recorded information of cell by modifying the data structure of the literature [5], and the necessary information in cell for each mobile is shown in Table 2. The method on updating the information is the same as [5]. Therefore the accumulation function can be defined as (1), where the weight factors $w_{-} o f \_k, w_{-} o f_{-} t$ and $w_{-} o f_{-} p$ are represented weight of relational function, weight of the average dwell time and weight of movement probability, respectively. The weight of each factor stands for the dominant portion for the handoff and can be adjusted dynamically according to the real situation.

$$
A(w)=k(v) * w_{-} o f \_k+w_{-} o f_{-} t+w_{-} o f \_m .
$$

\section{Handoff Algorithm and Simulation}

Owing to the evolution of cellular networks, the cell structure of diameter size becomes small. If the frequencies of handoff occurring are increasing, the QoS will be faced with strict test. Once the QoS is not guaranteed, the quality of call may be lowered, or the CDP may increase. For such a reason, there have been many researches that concentrated on their attention for channel allocation and reduced the CDP. In the literatures, the prioritized channel allocation for handoff can be classified into two classes as follows: one is queuing handoff requests and the other is reserving a number of channels exclusively for handoff requests. Briefly, the two kinds of channel allocation will bring on decreasing handoff failure and increasing CBP that will make the utilization of channel no efficiency in turn. 


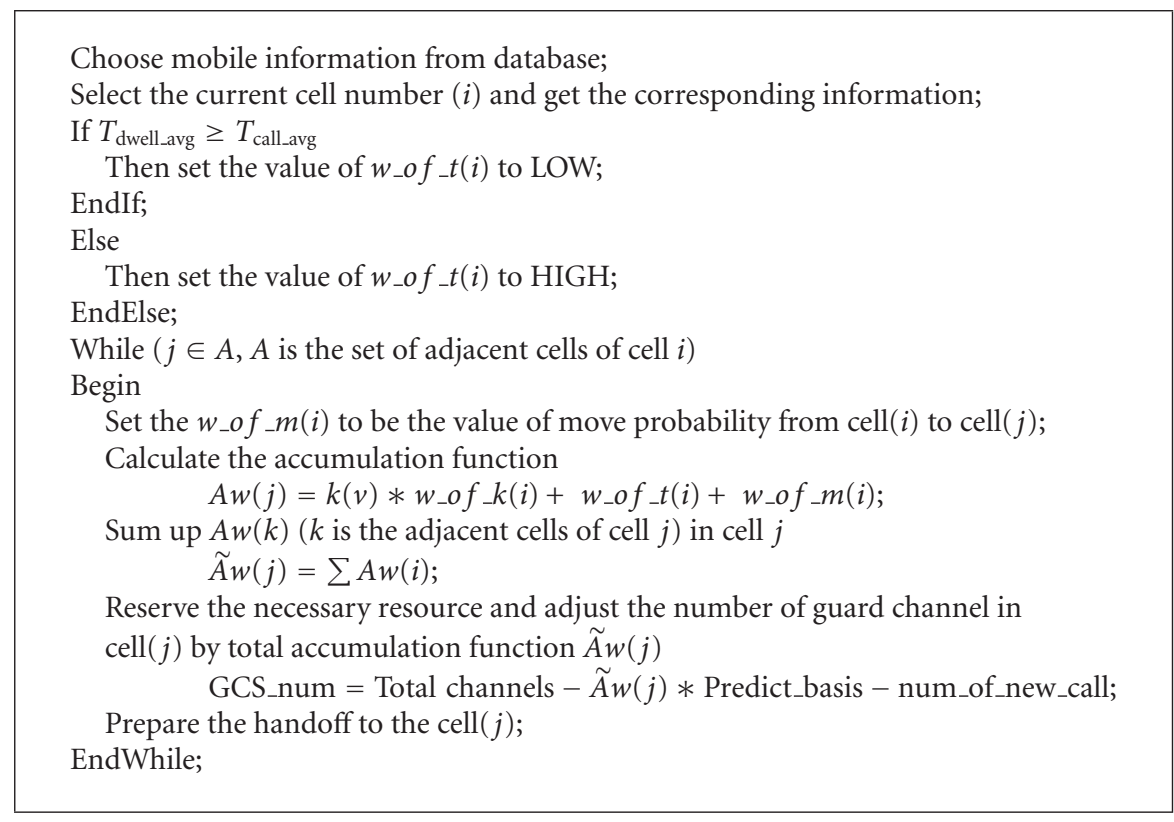

Algorithm 1

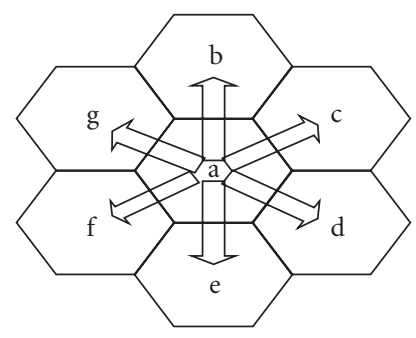

Figure 1: Possible cells of handoff call.

Based on the relative literature, many channel allocation schemes were mentioned, such as fully shared scheme (FSS), guard channel scheme (GCS), and so on. For the FSS, the call requests are not distinguished handoff calls from new calls for base station. Owing to all of the available channels in a base station shared by new calls and handoff calls, the number of new call admission can be maximized, and then the utilization of wireless channels will be more effective. However, it is difficult to guarantee the QoS for handoff call, which CDP will increase. Furthermore, if the handoff call can be predicted before, the CDP will be decreased by assigning the necessary channels in cells that handoffs will occur in future. In opposition to FSS, some literatures $[5,8]$ about GCS, which are exclusively reserved for handoff calls, called guard channels, and the remaining channels, called shared channels that can be shared equally between handoff calls and new calls, were proposed for handoff call channel allocation. Obviously, once the normal channels are exhausted by new calls and handoff calls, the GCS rejects new calls until the available channels go below threshold. However, it is a tradeoff between handoff calls and new calls to select an appropriate threshold for the number of guard channels. Thus, if it is possible to predict handoff calls adequately, the required channels of handoff calls can be reserved beforehand and the number of guard channels can be reduced. To over such a problem, the proposed scheme is not only to predict the handoff calls but also to adjust the number of guard channels dynamically by adopting the accumulation function value.

The major purpose of handoff algorithm is to keep the QoS above a certain level or prevent call dropping for being short of the necessary resource. The proposed scheme considers user constant routes and data structure of each cell for each mobile by modifying the data structure of reference research [5]. The relative design and update for cell information are omitted, so the design issues of the proposed scheme will be only the enhanced ones. After getting information and confirming the current cell number from database, the value of $w_{-} o f_{\_} t$ is set by comparing $T_{\text {dwell_avg }}$ with $T_{\text {call_avg }}$, and the values will be set either " $L O W$ " or " $H I G H$ ", where " $L O W$ " and "HIGH" are adjustable definition constants. Moreover, the calculation of accumulation function value in current cell triggers off the calculations of total accumulation function value in adjacent cells, and the total accumulation function value can be regarded as the factor for adjusting the number of channels in the six adjacent cells, where "Predict_basis" and "num_of_new_call" are the initial values for each cell and adjustable for the real situation. The most difference among the proposed scheme and the literatures $[5,8]$ is the adaptive threshold for the number of guard channels by using the total accumulation function number " $\tilde{A} w(j)$ ", and the proposed scheme is described exhaustively as in Algorithm 1 .

For performance evaluations, the terms of dropping probability of handoff calls, blocking probability of a new call, and channel utilization are compared between predicted handoff and unpredicted handoff. The number of mobiles is 


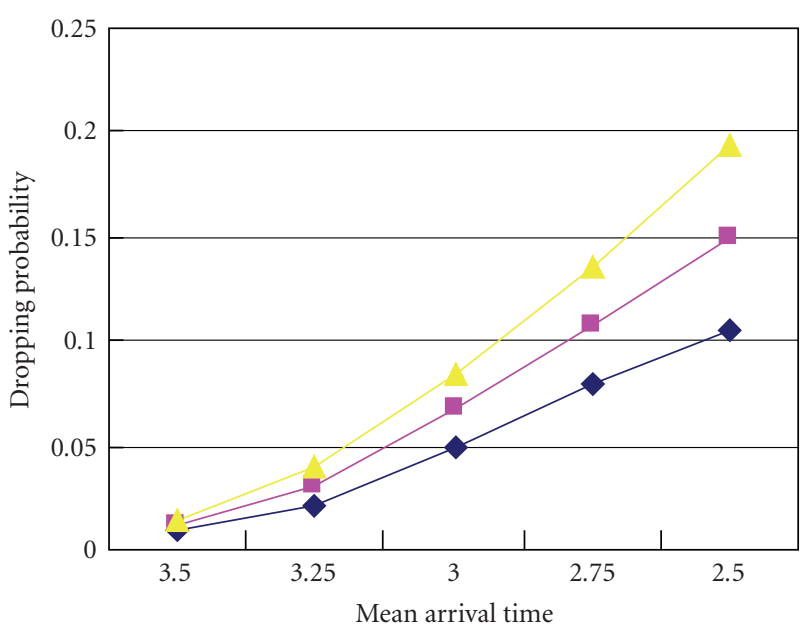

(a) Dropping probability

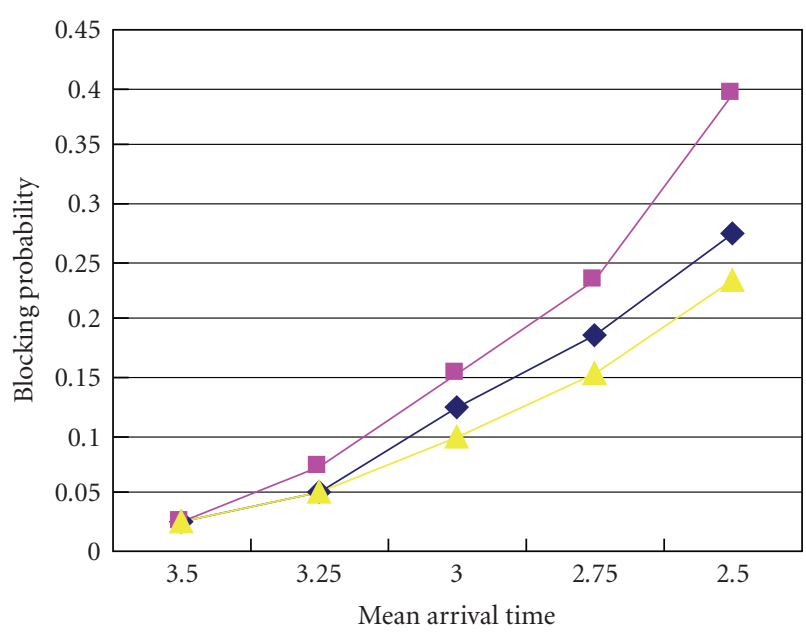

(b) Blocking probability

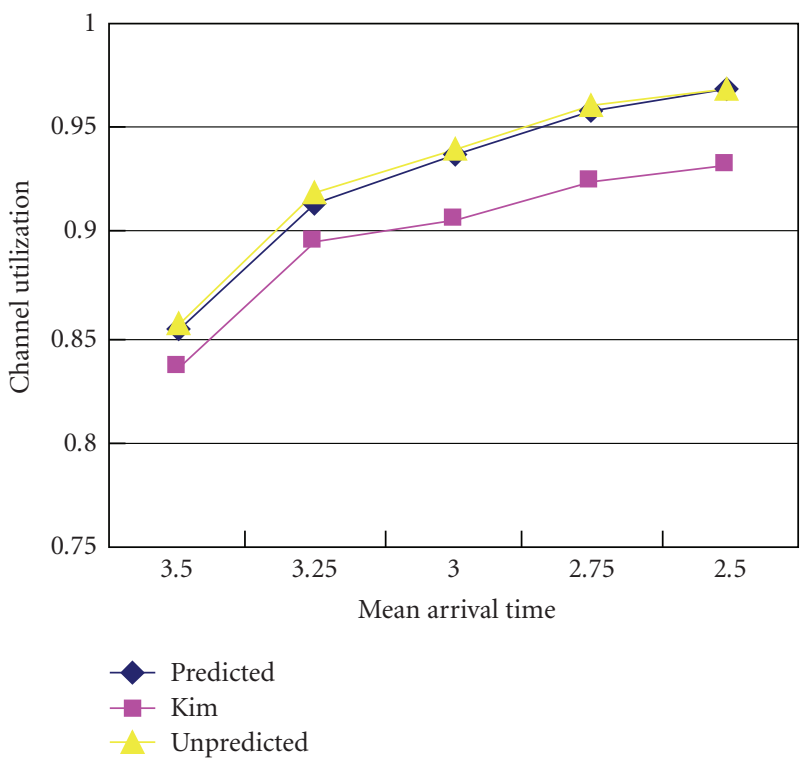

(c) Channel utilization

Figure 2: Simulation results of handoff prediction. bigger than the channels in cell so that the traffic model to the cell can be approximated as a Poisson process $[8,9]$. The related parameters are shown as follows:

(i) $\lambda$ : the call arrival rate according to Poisson process of rate $\lambda[8,9]$.

(ii) $\mu$ : the mean completion time assumed to exponential distribution with mean of $1 / \mu$ [9].

(iii) $\theta$ : the portable mobility and the dwell time is assumed to be exponential distribution with mean of $1 / \theta[9]$.

In our experiments, the mean of call holding time $1 / \mu$ is 4 minutes, and the mean of dwell time $1 / \theta$ is 2 minutes. According to the assumptions of experiments, the handoff calls are $50 \%$ of total calls, and predicted handoff calls are $50 \%$ of handoff calls. The simulation results which are the comparisons among the proposed scheme, the similar concept that considers user mobility proposed by Kim and Jung [5], and the unpredicted scheme, respectively, are shown in Figure 2. The proposed scheme is with lower CDP than Kim's and the unpredicted one, and the CBP of the proposed scheme is also lower than Kim's scheme and only a little higher than unpredicted one. Besides, the proposed scheme almost can achieve the same channel utilization as the unpredicted one, but, Kim's cannot achieve the same performance in term of utilization. Briefly, the proposed scheme has better performances in term of CDP and CBP than Kim's, almost achieves the same utilization as the unpredicted one.

\section{Conclusions and Future Works}

In this paper, a new scheme for handoff prediction was proposed, and the Extenics was also introduced and applied to transferring the speed of the mobile movement into the relational function. The accumulation function that is derived from the speed of movement, dwell time, and move probability is applied for adjusting the number of guard channels dynamically. The proposed scheme reserves the necessary number of channels for handoff calls in normal channel and reduces the number of guard channels, hence, the CDP can be decrease and QoS is also guaranteed.

Due to scarce resource of wireless bandwidth, the extension and accumulation concepts are applied to predicting handoff call and adjust the number of guard channels. If the number of guard channels is low, there will be more new calls admitted so that the channel utilization will increase. In the experiment results, the utilization is almost equal between predicted and unpredicted. Furthermore, we will try to compare the number of guard channels with others in the future.

\section{Acknowledgment}

This work was supported in part by the National Science Council of Taiwan under the Grants NSC97-2221-E-412001. 


\section{References}

[1] J. Misic and T. Y. Bun, "On call level QoS guarantees under heterogeneous user mobilities in wireless multimedia networks," in Proceedings of IEEE Global Telecommunication Conference (GLOBECOM '99), vol. 5, pp. 2730-2736, Rio de Janeiro, Brazil, December 1999.

[2] K. L. Yeung and S. Nanda, "Channel management in microcell/macrocell cellular radio systems," IEEE Transactions on Vehicular Technology, vol. 45, no. 4, pp. 601-612, 1996.

[3] A. Gurijala and C. Molina, "Defining and monitoring QoS metrics in the next generation wireless networks," in Proceedings of IEE Telecommunications Quality of Services: The Business of Success (QqS '04), pp. 37-42, London, UK, March 2004.

[4] Y. Fang and I. Chlamtac, "Teletraffic analysis and mobility modeling of PCS networks," IEEE Transactions on Communications, vol. 47, no. 7, pp. 1062-1072, 1999.

[5] H. K. Kim and J.-I. Jung, "The handover algorithm that considers the user's mobility pattern in wireless ATM," IEICE Transactions on Fundamentals of Electronics, Communications and Computer Sciences, vol. E84-A, no. 6, pp. 1362-1369, 2001.

[6] H. Chen, S. Kumar, and C.-C. J. Kuo, "Dynamic call admission control scheme for QoS priority handoff in multimedia cellular system," in Proceedings of IEEE Wireless Communications and Networking Conference (WCNC'02), vol. 1, pp. 114-118, March 2002.

[7] W. Cai, "Extension theory and its application," Chinese Science Bulletin, vol. 44, no. 17, pp. 1538-1548, 1999.

[8] Y. C. Kim, D. E. Lee, B. J. Lee, Y. S. Kim, and B. Mukherjee, "Dynamic channel reservation based on mobility in wireless ATM networks," IEEE Communications Magazine, vol. 37, no. 11, pp. 47-51, 1999.

[9] Y.-B. Lin, S. Mohan, and A. Noerpel, "PCS channel assignment strategies for hand-off and initial access," IEEE Personal Communications, vol. 1, no. 3, pp. 47-56, 1994. 

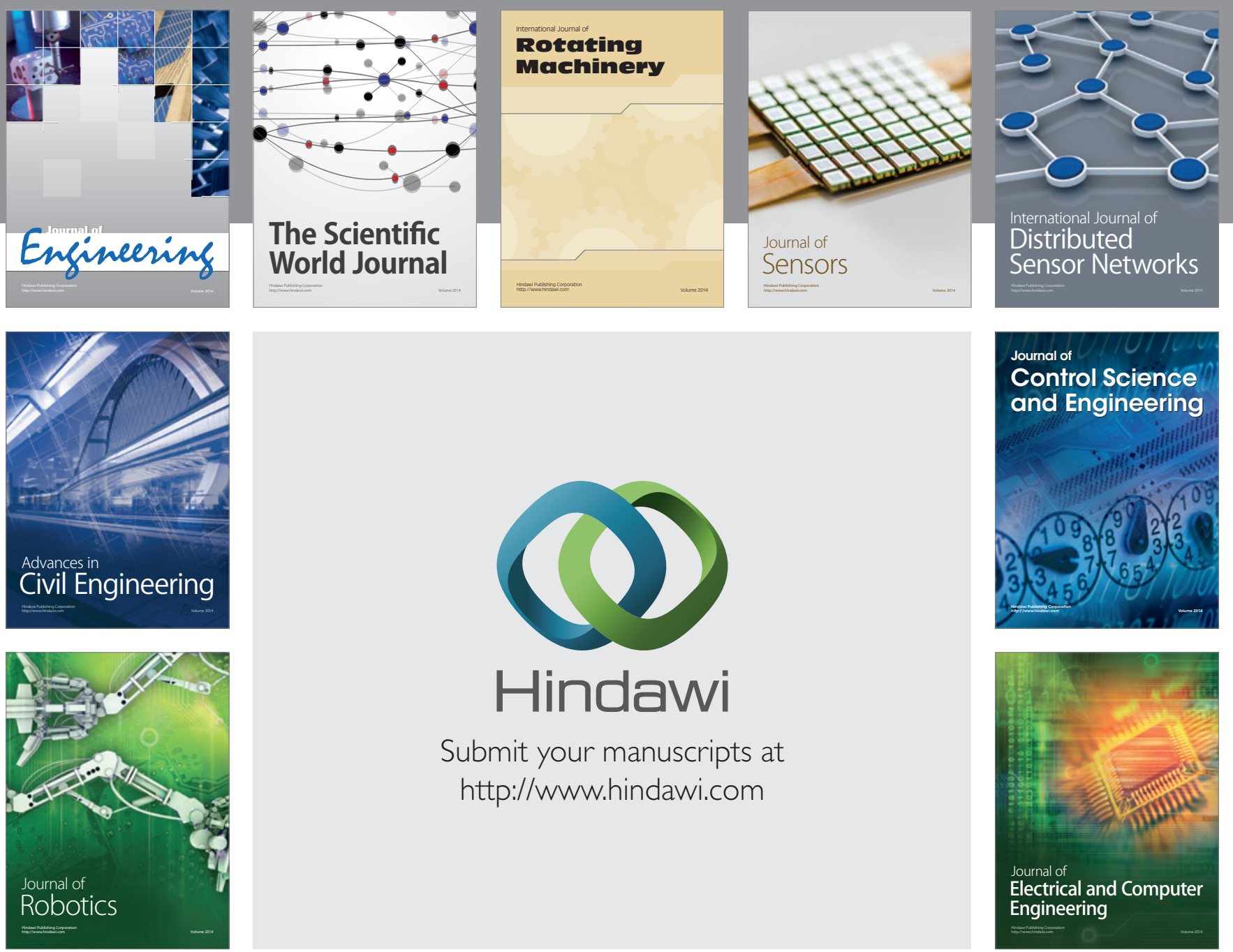

Submit your manuscripts at

http://www.hindawi.com
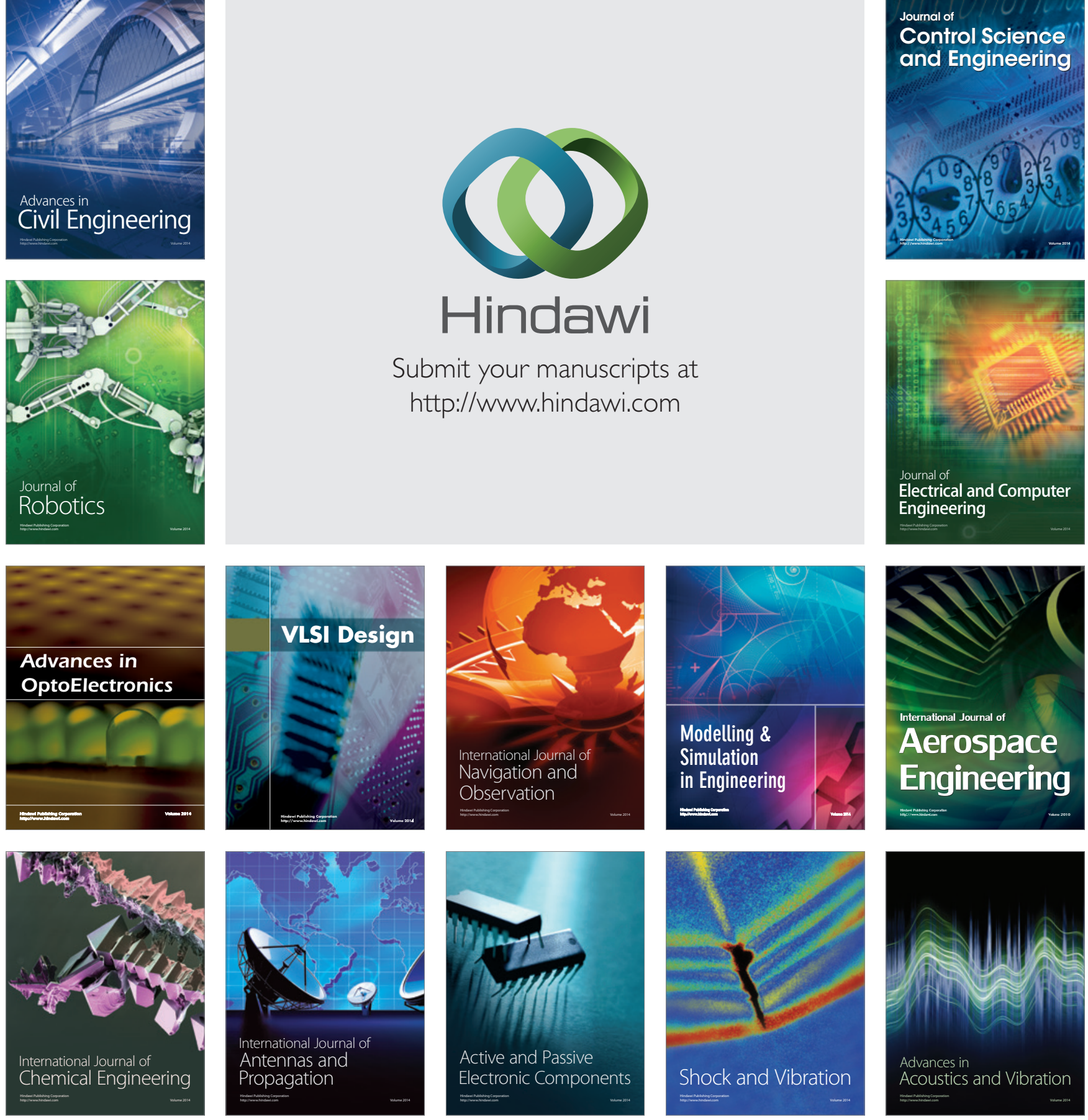